\title{
Entkopplungsregelungen
}

\section{für lineare überaktuierte Systeme}

\author{
Non-Interacting Control for Over-Actuated Linear Systems
}

\author{
Arne Wahrburg*, Jürgen Adamy, Technische Universität Darmstadt \\ * Korrespondenzautor: wahrburg@rtr.tu-darmstadt.de
}

Zusammenfassung Gegenstand des Beitrags ist der Entwurf von statischen Zustandsreglern zur Führungsentkopplung in linearen überaktuierten Systemen. Zum einen wird eine Erweiterung der Entwurfsmethodik nach Falb und Wolovich erläutert, welche eine einfache Nutzung der zusätzlichen Freiheitsgrade gegenüber klassischen Entkopplungsreglern ermöglicht. Zum anderen erlaubt ein parametrischer Entwurf die Interpretation der entstehenden Freiheitsgrade. Als Anwendungsbeispiele werden die Reduzierung der Stellenergie sowie die Steigerung der Robustheit von Entkopplungsregelungen vorgestellt.

\begin{abstract}
$\leadsto \quad$ Summary This paper presents design approaches for non-interacting controllers for over-actuated linear systems. Two design schemes are presented. An extension of the well known design by Falb and Wolovich enables a straightforward exploitation of the additional degrees of freedom compared to standard non-interacting controllers. Furthermore, a parametric approach is presented, which allows a comprehensible interpretation of the resulting degrees of freedom. The design is applied to reduce control effort as well as to increase robustness of non-interacting controllers.
\end{abstract}

Schlagwörter Entkopplungsregler, überaktuierte Systeme, parametrischer Entwurf, energieeffiziente Regelung, robuste Entkopplung, lineare Matrixungleichungen $\leadsto$ Keywords Non-interacting controllers, over-actuated systems, parametric design, energy-efficient control, robust decoupling, linear matrix inequalities

\section{Einleitung}

Der Entwurf von Entkopplungsregelungen für lineare zeitinvariante Systeme ist ein seit geraumer Zeit intensiv studiertes Gebiet der Regelungstechnik. Unter dem Begriff der Führungsentkopplung wird die Diagonalisierung der Übertragungsmatrix des geschlossenen Regelkreises verstanden. Dies erlaubt die gezielte Regelung einer einzelnen Ausgangsgröße, da sie lediglich von einer ihr zugeordneten Führungsgröße beeinflusst wird.

Der Entwurf von statischen Zustandsreglern zur Führungsentkopplung geht zurück auf [24]. Für Systeme mit ebenso vielen Eingangs- wie Ausgangsgrößen (so genannte quadratische Systeme) finden sich in [9] die bekannten notwendigen und hinreichenden Bedingungen für die Entkoppelbarkeit eines Systems sowie Entwurfsgleichungen. Ein äquivalentes Kriterium sowie ein alternatives, zustandsraumbasiertes Entwurfsverfahren ist in [28] gegeben. Es beruht auf der Vollständigen Modalen
Synthese [27] und erlaubt insbesondere bei Systemen mit Invarianten Nullstellen einen transparenteren Entwurf.

Nicht-quadratische Systeme mit mehr Eingangs- als Ausgangsgrößen werden auch als überaktuierte Systeme bezeichnet. Häufig sind solche Systeme in Luftfahrtanwendungen anzutreffen [25], da sie aufgrund der vorhandenen Redundanz einerseits Sicherheitsreserven bereitstellen und andererseits die Manövrierbarkeit erhöhen. Es stellt sich dann die Frage, wie das $\mathrm{Zu}$ sammenwirken der Aktoren im Sinne unterschiedlicher Güteanforderungen gestaltet wird. Ein Überblick zu dieser sogenannten control allocation findet sich u. a. in [25] und [35]. In jüngerer Vergangenheit wurden zahlreiche Ergebnisse publiziert, die z. T. auch eine dynamische Allokation beinhalten. So wird in [10] das Regulatorproblem für lineare überaktuierte Systeme gelöst, während in [1] und [4] eine möglichst energieeffiziente Regelung angestrebt wird. Auch für Systeme mit Stellgrößen- 
begrenzungen [15] und eingangs-affine Systeme [12] existieren bereits Ergebnisse.

Dieser Beitrag befasst sich mit der Entkopplungsregelung von linearen überaktuierten Systemen. Die betrachteten Systeme verfügen über $n_{u}$ Eingangsgrößen, mit denen $n_{y}<n_{u}$ Ausgangsgrößen geregelt werden. Für diesen Fall existieren bisher noch keine allgemeingültigen notwendigen und hinreichenden Bedingungen für die Entkoppelbarkeit linearer Systeme [8;11]. Auch aktuelle Resultate beschränken sich entweder auf hinreichende Bedingungen oder aber eingeschränkte Systemklassen $[30 ; 34]$. Auch dieser Beitrag stützt sich auf eine hinreichende Bedingung. Es wird vorausgesetzt, dass es unter den $n_{u}$ Aktoren eine Gruppe von $n_{y}$ Aktoren gibt, mit denen das entsprechende quadratische System entkoppelt werden kann. Dementsprechend ist die Existenz eines entkoppelnden statischen Zustandsreglers für das überaktuierte System gewährleistet. Es werden zwei Entwurfsverfahren vorgestellt, welche jeweils als Erweiterungen der Ergebnisse aus [9] bzw. [28] verstanden werden können. Dabei ergeben sich gegenüber den klassischen Resultaten zusätzliche Freiheitsgrade im Entwurf. Diese werden im vorliegenden Artikel für zwei unterschiedliche Problemstellungen genutzt. Zum einen wird die aufzuwendende Stellenergie minimiert, zum anderen wird die Robustheit bezüglich unsicherer Modellparameter betrachtet. Beide Problemstellungen werden auf lineare Matrixungleichungen (LMIs) zurückgeführt.

Dementsprechend gliedert sich der Beitrag wie folgt. Nach Einführung der verwendeten Notation und einigen grundlegenden Betrachtungen erfolgt in Abschnitt 3 der allgemeine Entwurf von Entkopplungsregelungen für überaktuierte Systeme. In Abschnitt 4 werden die Entwurfsverfahren zur Optimierung der Stellenergie und zur Steigerung der Robustheit angewendet. Beispielhaft werden die Resultate in Abschnitt 5 für ein linearisiertes Flugzeugmodell angewendet.

\section{Grundlagen}

\subsection{Notation}

Das Spektrum einer Matrix $\boldsymbol{A} \in \mathbb{R}^{n \times n}$ wird mit $\sigma(\boldsymbol{A})$ bezeichnet, für positiv (negativ) definite Matrizen wird $\boldsymbol{A} \succ(\prec) \mathbf{0}$ geschrieben. Eine Matrix $\boldsymbol{P} \in \mathbb{R}^{m \times n}$ mit $n>m$ und $\operatorname{rang}(\boldsymbol{P})=m$ ist rechtsinvertierbar, d.h. $\boldsymbol{P} \boldsymbol{P}^{+}=\boldsymbol{I}_{m}$, wobei $\boldsymbol{P}^{+} \in \mathbb{R}^{n \times m}$ die Pseudoinverse ist. Weiterhin gilt der Rangsatz [22]

Lemma 1 (Rank-Nullity-Theorem [22]). Für eine Matrix $\boldsymbol{P} \in \mathbb{R}^{m \times n}$ gilt

$\operatorname{rang}(\boldsymbol{P})+\operatorname{dim}(\operatorname{ker}(\boldsymbol{P}))=n$.

Dabei ist $\operatorname{dim}(\operatorname{ker}(\boldsymbol{P}))$ die Dimension des Nullraumes von $\boldsymbol{P}$ und es existiert wegen $\operatorname{rang}(\boldsymbol{P})=m$ ein orthogonales Komplement $\boldsymbol{P}^{\perp} \in \mathbb{R}^{n \times(n-m)}$, für welches $\boldsymbol{P} \boldsymbol{P}^{\perp}=\mathbf{0}$ gilt. Im Sinne einer kompakten Notation wird $\mathrm{He}(\boldsymbol{P})=\boldsymbol{P}+\boldsymbol{P}^{H}$ geschrieben, was sich für reelle Matrizen zu He $(\boldsymbol{P})=\boldsymbol{P}+\boldsymbol{P}^{\top}$ vereinfacht. Mit $\boldsymbol{p}_{i}^{\top}$ wird die $i$-te Zeile von $\boldsymbol{P}$ bezeichnet. In einer symmetrischen Matrix $\boldsymbol{P}=\boldsymbol{P}^{\top}$ bezeichnet das Symbol * Elemente, die sich aus Symmetriegründen ergeben. Die gezielte Ausnutzung der im Entwurf auftretenden zusätzlichen Freiheitsgrade greift auf das Bounded-Real-Lemma [3] zurück.

Lemma 2 (Bounded-Real-Lemma [3]). Gegeben sei ein stabiles, vollständig steuerbares, lineares zeitinvariantes System $\dot{\boldsymbol{x}}=\boldsymbol{A} \boldsymbol{x}+\boldsymbol{B} \boldsymbol{u}, \boldsymbol{y}=\boldsymbol{C} \boldsymbol{x}+\boldsymbol{D} \boldsymbol{u}$ mit der Übertragungsmatrix $\boldsymbol{G}(s)=\boldsymbol{C}(s \boldsymbol{I}-\boldsymbol{A})^{-1} \boldsymbol{B}+\boldsymbol{D}$. Dann gilt $\|\boldsymbol{G}(s)\|_{\infty}<\gamma$ genau dann, wenn eine reelle symmetrische Matrix $\boldsymbol{X} \succ \mathbf{0}$ existiert, so dass

$$
\left[\begin{array}{ccc}
\operatorname{He}(\boldsymbol{X} \boldsymbol{A}) & \boldsymbol{X} \boldsymbol{B} & \boldsymbol{C}^{\top} \\
* & -\gamma \boldsymbol{I} & \boldsymbol{D}^{\top} \\
* & * & -\gamma \boldsymbol{I}
\end{array}\right] \prec \mathbf{0} .
$$

\subsection{Problemstellung}

Betrachtet werden vollständig steuerbare lineare zeitinvariante Systeme der Form

$\dot{x}=A x+B u$,

$y=C x+D u$,

wobei $\boldsymbol{x} \in \mathbb{R}^{n}, \boldsymbol{u} \in \mathbb{R}^{n_{\mathcal{u}}}$ und $\boldsymbol{y} \in \mathbb{R}^{n_{y}}$ gilt und die Matrizen jeweils entsprechende Dimensionen aufweisen. Dabei wird $\operatorname{rang}(\boldsymbol{C})=n_{y}$ und $n_{y} \leq \operatorname{rang}(\boldsymbol{B}) \leq n_{u}$ angenommen. Das System (1) wird abkürzend als $(\boldsymbol{A}, \boldsymbol{B}, \boldsymbol{C}, \boldsymbol{D})$ geschrieben. Es soll mittels eines statischen linearen $\mathrm{Zu}$ standsreglers

$u=-K x+F w$

so beeinflusst werden, dass die Übertragungsmatrix $\boldsymbol{G}_{\boldsymbol{y} \boldsymbol{w}}(s)$, welche die Führungsgrößen $\boldsymbol{w} \in \mathbb{R}^{n_{y}}$ mit den Ausgangsgrößen $y$ verknüpft, Diagonalgestalt aufweist. Es soll also

$$
\begin{aligned}
\boldsymbol{G}_{\boldsymbol{y} \boldsymbol{w}}(s) & =(\boldsymbol{C}-\boldsymbol{D} \boldsymbol{K})\left(s \boldsymbol{I}_{n}-(\boldsymbol{A}-\boldsymbol{B} \boldsymbol{K})\right)^{-1} \boldsymbol{B} \boldsymbol{F}+\boldsymbol{D} \boldsymbol{F} \\
& =\operatorname{diag}\left(g_{1,1}(s), \ldots, g_{n_{y}, n_{y}}(s)\right)
\end{aligned}
$$

gelten. Weiterhin ist die Stabilität des geschlossenen Regelkreises sicherzustellen, d.h. die Matrix $\boldsymbol{A}-\boldsymbol{B} \boldsymbol{K}$ muss eine Hurwitz-Matrix sein.

Die Diagonalelemente der Führungsübertragungsmatrix werden wie im quadratisch Fall [28] angesetzt als

$g_{i, i}(s)=\frac{z_{i, 0}}{s^{\delta_{i}}+q_{i, \delta_{i}-1} \delta^{\delta_{i}-1}+\ldots+q_{i, 1} s+q_{i, 0}}$.

Darin sind $\delta_{i}$ die Differenzenordnungen der einzelnen Ausgänge. Sie beschreiben, wie oft ein Ausgang zeitlich differenziert werden muss, bis der Eingang $\boldsymbol{u}$ explizit auftaucht. Entsprechend sind sie definiert als

$\delta_{i}= \begin{cases}0, & \boldsymbol{d}_{i}^{\top} \neq \mathbf{0}^{\top}, \\ \min _{k}\left\{k \geq 1: \boldsymbol{c}_{i}^{\top} \boldsymbol{A}^{k-1} \boldsymbol{B} \neq \mathbf{0}^{\top}\right\}, & \boldsymbol{d}_{i}^{\top}=\mathbf{0}^{\top},\end{cases}$ 
wobei $\boldsymbol{d}_{i}^{\top}$ die $i$-te Zeile der Durchgriffsmatrix $\boldsymbol{D}$ ist. Daraus ergibt sich die Differenzordnung des Systems, welche mittels

$\delta=\sum_{i=1}^{n_{y}} \delta_{i}$

bestimmt wird. Für Systeme mit Durchgriff ergibt sich die Entkoppelbarkeitsmatrix in Anlehnung an [9] zu

$\boldsymbol{D}^{*}=\left[\begin{array}{c}\boldsymbol{d}_{1}^{* \top} \\ \vdots \\ \boldsymbol{d}_{n_{y}}^{* \top}\end{array}\right], \boldsymbol{d}_{i}^{* \top}= \begin{cases}\boldsymbol{d}_{i}^{\top}, & \delta_{i}=0, \\ \boldsymbol{c}_{i}^{\top} \boldsymbol{A}^{\delta_{i}-1} \boldsymbol{B}, & \delta_{i} \geq 1 .\end{cases}$

In diesem Beitrag wird stets vorausgesetzt, dass die folgende Annahme gilt.

Annahme 1. Die Matrix $D^{*}$ hat vollen Zeilenrang und ist somit rechtsinvertierbar.

In Abschnitt 3.1 wird gezeigt, dass diese Annahme die Existenz einer entkoppelnden Regelung gewährleistet. Geht man wie in der Einleitung erwähnt davon aus, dass eine Gruppe von $n_{y}$ Aktoren existiert, mit denen das entsprechende quadratische System entkoppelt werden kann, so ist Annahme 1 erfüllt.

Um stabile Entkoppelbarkeit zu gewährleisten, wird darüber hinaus die folgende Annahme vorausgesetzt.

Annahme 2. Das System (1) weist keine Invarianten Nullstellen in der geschlossenen rechten s-Halbebene auf.

\section{Reglerentwurf}

Im folgenden Abschnitt werden zwei Entwurfsverfahren vorgestellt, welche die Entkopplung überaktuierter Systeme der Form (1) erlauben.

\subsection{Erweiterung des Entwurfes nach Falb und Wolovich}

Das erste vorgestellte Entwurfsverfahren zur Entkopplung nicht-quadratischer linearer Systeme stellt eine Erweiterung der Entwurfsmethodik von Falb und Wolovich [9] dar und ist im folgenden Satz zusammengefasst.

Satz 1. Gegeben sei ein System der Form (1), welches die Differenzenordnungen $\delta_{i}$ und eine rechtsinvertierbare Matrix $D^{*}$ aufweist. Dann erzeugen alle statischen Zustandsregler $\boldsymbol{K}=\boldsymbol{K}_{0}+\boldsymbol{D}^{* \perp} \boldsymbol{R}_{K}$ mit dem zugehörigen Vorfilter $\boldsymbol{F}=\boldsymbol{F}_{0}+\boldsymbol{D}^{* \perp} \boldsymbol{R}_{F}$, welche durch beliebige Matrizen $\boldsymbol{R}_{K} \in \mathbb{R}^{\left(n_{u}-n_{y}\right) \times n}$ und $\boldsymbol{R}_{F} \in \mathbb{R}^{\left(n_{u}-n_{y}\right) \times n_{y}}$ sowie $\boldsymbol{K}_{0}=\boldsymbol{D}^{*+} \boldsymbol{M}$, $\boldsymbol{F}_{0}=\boldsymbol{D}^{*+} \boldsymbol{N}$ mit

$\boldsymbol{M}=\left[\begin{array}{c}\boldsymbol{c}_{1}^{\top}\left(\boldsymbol{A}^{\delta_{1}}+\sum_{k=0}^{\delta_{1}-1} q_{1, k} \boldsymbol{A}^{k}\right) \\ \vdots \\ \boldsymbol{c}_{n_{y}}^{\top}\left(\boldsymbol{A}^{\delta_{n_{y}}+\sum_{k=0}^{\delta_{n_{y}}-1}} q_{n_{y}, k} \boldsymbol{A}^{k}\right)\end{array}\right]$,

$N=\operatorname{diag}\left(z_{1,0}, \ldots, z_{n_{y}, 0}\right)$ gegeben sind, eine diagonale Übertragungsmatrix $\mathbf{G}_{\boldsymbol{y} w}(s)=$ $\operatorname{diag}\left(g_{1,1}(s), \ldots, g_{n_{y}, n_{y}}(s)\right)$ mit

$g_{i, i}(s)=\frac{z_{i, 0}}{s^{\delta_{i}}+q_{i, \delta_{i}-1} \delta^{\delta_{i}-1}+\ldots+q_{i, 0}}$.

Beweis 1. Da $D^{*}$ nach Annahme 1 rechtsinvertierbar ist, gilt $\operatorname{rang}\left(\boldsymbol{D}^{*}\right)=n_{y}$. Wegen $\boldsymbol{D}^{*} \in \mathbb{R}^{n_{y} \times n_{u}}$ folgt demnach aus Lemma $1 \boldsymbol{D}^{* \perp} \in \mathbb{R}^{n_{u} \times\left(n_{u}-n_{y}\right)}$. Für Ausgänge mit Durchgriff, d. h. $\delta_{i}=0$, gilt

$y_{i}=\boldsymbol{c}_{i}^{\top} \boldsymbol{x}+\boldsymbol{d}_{i}^{\top} \boldsymbol{u}$.

Für alle anderen Ausgänge ergeben sich die zeitlichen Ableitungen sukzessive zu

$y_{i}=\boldsymbol{c}_{i}^{\top} \boldsymbol{x}$,

$\dot{y}_{i}=\boldsymbol{c}_{i}^{\top} \boldsymbol{A x}$,

$y_{i}^{\left(\delta_{i}-1\right)}=\boldsymbol{c}_{i}^{\top} \boldsymbol{A}^{\delta_{i}-1} \boldsymbol{x}$,

$y^{\left(\delta_{i}\right)}=\boldsymbol{c}_{i}^{\top} \boldsymbol{A}^{\delta_{i}} \boldsymbol{x}+\boldsymbol{c}_{i}^{\top} \boldsymbol{A}^{\delta_{i}-1} \boldsymbol{B} \boldsymbol{u}$,

da nach (5) $\boldsymbol{c}_{i}^{\top} \boldsymbol{A}^{k} \boldsymbol{B}=\mathbf{0}^{\top} \forall k=0, \ldots, \delta_{i}-2$ ist. Im Zeitbereich lautet die vorgegebene Dynamik für die Übertragungspfade

$y_{i}^{\left(\delta_{i}\right)}+q_{i, \delta_{i}-1} y_{i}^{\left(\delta_{i}-1\right)}+\ldots+q_{i, 0} y_{i}=z_{i, 0} w_{i}$.

Setzt man (8) bzw. (9) in (10) ein, so ergibt sich

$\boldsymbol{c}_{i}^{\top}\left(\boldsymbol{A}^{\delta_{i}}+\sum_{k=0}^{\delta_{i}-1} q_{i, k} \boldsymbol{A}^{k}\right) \boldsymbol{x}+\underbrace{\boldsymbol{c}_{i}^{\top} \boldsymbol{A}^{\delta_{i}-1} \boldsymbol{B}}_{\boldsymbol{d}_{i}^{* \top}} \boldsymbol{u}=z_{i, 0} w_{i}$.

Da nach (7) $\boldsymbol{d}_{i}^{* \top}=\boldsymbol{d}_{i}^{\top}$ für Ausgänge mit Durchgriff gilt, lässt sich (11) mit den Matrizen $M$ und $N$ aus Satz 1 für alle Ausgänge zusammenfassen zu der Bedingung

$D^{*} u=-M x+N w$.

Für den in Satz 1 angegebenen Regler und Vorfilter lautet das Regelgesetz

$\boldsymbol{u}=-\left(D^{*+} \boldsymbol{M}+D^{* \perp} \boldsymbol{R}_{K}\right) \boldsymbol{x}+\left(D^{*+} N+D^{* \perp} \boldsymbol{R}_{F}\right) \boldsymbol{w}$.

Durch Einsetzen in (12) ergibt sich wegen $\boldsymbol{D}^{*} \boldsymbol{D}^{*+}=\boldsymbol{I}_{n_{y}}$ und $D D^{* \perp}=\mathbf{0}$, dass die Entkopplungsbedingung (12) für beliebige $\boldsymbol{R}_{K}$ und $\boldsymbol{R}_{F}$ erfüllt ist.

Gegenüber dem klassischen Entwurfsverfahren, bei dem lediglich die Koeffizienten $z_{i, 0}$ sowie $q_{i, j}$ als Freiheitsgrade verbleiben, stehen im überaktuierten Fall also noch die beliebigen Matrizen $\boldsymbol{R}_{K}$ und $\boldsymbol{R}_{F}$ als freie Parameter zur Verfügung. Im letzten Schritt des Beweises 1 zeigt sich, dass Annahme 1 eine hinreichende Bedingung für die Entkoppelbarkeit des überaktuierten Systems ist. Es sei an dieser Stelle jedoch angemerkt, dass diese Bedingung nicht notwendig ist (s. z. B. [8]). Außerdem ist zu beachten, dass für $\delta<n$ im geschlossenen Kreis unbeobachtbare Eigenwerte auftreten, die je nach Wahl von $\boldsymbol{R}_{K}$ instabil 
sein können. Außerdem können auch Eigenwerte auftreten, die invariant bezüglich der Wahl von $\boldsymbol{R}_{K}$ sind. Das im folgenden Abschnitt gezeigte Verfahren ist diesbezüglich wesentlich transparenter.

\subsection{Parametrischer Entwurf}

Alternativ zum Verfahren aus Abschnitt 3.1 wird im Folgenden ein Entwurf auf Basis der Vollständigen Modalen Synthese [27] vorgestellt, welcher eine anschauliche Interpretation der zusätzlichen Freiheitsgrade erlaubt. Er ist als Erweiterung der Ergebnisse in [18-20] und [28] zu verstehen.

Im Sinne einer übersichtlichen Darstellung werden die beiden folgenden Annahmen getroffen.

Annahme 3. Die Eigenwerte des geschlossenen Regelkreises sind paarweise verschieden, d.h. $\lambda_{R_{i}} \neq \lambda_{R_{j}}$ $\forall \lambda_{R_{i}}, \lambda_{R_{j}} \in \sigma(\boldsymbol{A}-\boldsymbol{B} \boldsymbol{K})$.

Annahme 4. Kein Eigenwert des geschlossenen Regelkreises fällt mit einem Eigenwert des offenen Kreises zusammen, d. h. $\lambda_{R_{i}} \notin \sigma(\boldsymbol{A}) \forall \lambda_{R_{i}} \in \sigma(\boldsymbol{A}-\boldsymbol{B K})$.

Beide Annahmen stellen wie in [16] und [28] gezeigt keine Einschränkung der Allgemeinheit dar, da sie durch entsprechende Erweiterungen des Verfahrens umgangen werden können.

Im Weiteren werden die zum Eigenwert $\lambda_{R_{k}}$ gehörigen Rechtseigenvektoren mit $\boldsymbol{v}_{R_{k}}$ bezeichnet, die entsprechenden Linkseigenvektoren mit $\boldsymbol{w}_{R_{k}}^{\top}$. Die Vektoren $\boldsymbol{v}_{R_{k}}$ bilden die Spalten der Rechtseigenvektormatrix $V_{R}$, während $\boldsymbol{w}_{R_{k}}^{\top}$ die Zeilen der Linkseigenvektormatrix $\boldsymbol{W}_{R}=\boldsymbol{V}_{R}^{-1}$ darstellen. Weiterhin werden die in [27] eingeführten Parametervektoren $\mathbb{R}^{n_{u}} \ni \boldsymbol{p}_{k}=-\boldsymbol{K} \boldsymbol{v}_{R_{k}}$ definiert. Aufgrund von Annahme 3 lässt sich die Matrix $\left(s \boldsymbol{I}_{n}-(\boldsymbol{A}-\boldsymbol{B} \boldsymbol{K})\right)^{-1}$ diagonalisieren und (3) lässt sich schreiben als

$$
\begin{aligned}
\boldsymbol{G}_{\boldsymbol{y} \boldsymbol{w}}(s)= & (\boldsymbol{C}-\mathbf{D K}) \boldsymbol{V}_{R} \boldsymbol{W}_{R}\left(s \boldsymbol{I}_{n}-(\boldsymbol{A}-\boldsymbol{B} \boldsymbol{K})\right)^{-1} . \\
& \ldots \cdot \boldsymbol{V}_{R} \boldsymbol{W}_{R} \boldsymbol{B} \boldsymbol{F}+\boldsymbol{D} \boldsymbol{F} \\
= & (\boldsymbol{C}-\mathbf{D K}) \boldsymbol{V}_{R} \operatorname{diag}\left(\frac{1}{s-\lambda_{R_{1}}}, \ldots, \frac{1}{s-\lambda_{R_{n}}}\right) . \\
& \ldots \cdot \boldsymbol{W}_{R} \boldsymbol{B} \boldsymbol{F}+\boldsymbol{D F} \\
= & \sum_{k=1}^{n} \frac{(\boldsymbol{C}-\boldsymbol{D} \boldsymbol{K}) \boldsymbol{v}_{R_{k}} \cdot \boldsymbol{w}_{R_{k}}^{\top} \boldsymbol{B} \boldsymbol{F}}{s-\lambda_{R_{k}}}+\boldsymbol{D F} \\
= & \sum_{k=1}^{n} \frac{\left(\boldsymbol{C} \boldsymbol{v}_{R_{k}}+\boldsymbol{D} \boldsymbol{p}_{k}\right) \cdot \boldsymbol{w}_{R_{k}}^{\top} \boldsymbol{B} \boldsymbol{F}}{s-\lambda_{R_{k}}}+\boldsymbol{D F} .
\end{aligned}
$$

Im Weiteren werden die ersten $\delta$ Eigenwerte mittels der Parametervektoren jeweils definierten Zeilen in $\boldsymbol{G}_{\boldsymbol{y} \boldsymbol{w}}(s)$ zugewiesen. Dafür wird die Notation $\lambda_{R_{i j}}, i=1, \ldots, n_{y}$, $j=1, \ldots, \delta_{j}$ eingeführt. Der Eigenwert $\lambda_{R_{i j}}$ wird somit der $j$-te Pol aller Elemente der $i$-ten Zeile der Übertragungsmatrix $\boldsymbol{G}_{\boldsymbol{y} w}(s)$. Entsprechend wird auch die Notation $\boldsymbol{p}_{i j}$ eingeführt. Aufgrund des dyadischen Produktes $\left(\boldsymbol{C} \boldsymbol{v}_{R_{k}}+\right.$ $\left.\boldsymbol{D} \boldsymbol{p}_{k}\right) \cdot \boldsymbol{w}_{R_{k}}^{\top} \boldsymbol{B} \boldsymbol{F}$ erfolgt die Zuordnung des Eigenwertes $\lambda_{R_{i j}}$ zur $i$-ten Zeile von $G_{y w}(s)$ genau dann, wenn

$C \boldsymbol{v}_{R_{i j}}+D p_{i j}=\boldsymbol{e}_{i}$ gilt, wobei $\boldsymbol{e}_{i} \in \mathbb{R}^{n_{y}}$ in der $i$-ten Zeile das Element 1 enthält und sonst überall 0 . Aus der Definition der Rechtseigenvektoren des geschlossenen Kreises, $(\boldsymbol{A}-$ $\boldsymbol{B} \boldsymbol{K}) \boldsymbol{v}_{R_{k}}=\lambda_{R_{k}} \boldsymbol{v}_{R_{k}}$, folgt $\left(\lambda_{R_{k}} \boldsymbol{I}_{n}-\boldsymbol{A}\right) \boldsymbol{v}_{R_{k}}=-\boldsymbol{B} \boldsymbol{K} \boldsymbol{v}_{R_{k}}=\boldsymbol{B} \boldsymbol{p}_{k}$ und somit aufgrund von Annahme 4

$\boldsymbol{v}_{R_{i j}}=\left(\lambda_{R_{i j}} \boldsymbol{I}_{n}-\boldsymbol{A}\right)^{-1} \boldsymbol{B} \boldsymbol{p}_{i j}$.

Eingesetzt in (15) folgt daraus die Bedingung

$\underbrace{\left(C\left(\lambda_{R_{i j}} \boldsymbol{I}_{n}-\boldsymbol{A}\right)^{-1} \boldsymbol{B}+\boldsymbol{D}\right)}_{\boldsymbol{\Pi}_{i j}} \boldsymbol{p}_{i j}=\boldsymbol{e}_{i}$

mit $\Pi_{i j} \in \mathbb{R}^{n_{y} \times n_{u}}$. Diese Matrix ist rechtsinvertierbar, wenn der Regelungseigenwert so gewählt wird, dass $\lambda_{R_{i j}}$ keine Übertragungsnullstelle des offenen Kreises ist. Nach Lemma 1 existiert also ein orthogonales Komplement $\Pi_{i j}{ }^{\perp} \in \mathbb{R}^{n_{u} \times\left(n_{u}-n_{y}\right)}$ und alle Parametervektoren

$p_{i j}=\Pi_{i j}^{+} \boldsymbol{e}_{i}+\Pi_{i j}^{\perp} z_{i j}$

erfüllen die geforderte Bedingung (15). Darin ist $z_{i j} \in \mathbb{R}^{n_{u}-n_{y}}$ ein frei wählbarer Vektor, welcher zusätzliche Entwurfsfreiheitsgrade bietet. Er erlaubt es, die Parametervektoren innerhalb des von $\Pi_{i j}^{\perp}$ aufgespannten Unterraumes zu beeinflussen. Die zugehörigen Rechtseigenvektoren sind dann durch (16) gegeben.

Die verbleibenden $n-\delta$ Eigenwerte $\lambda_{R_{k}}$ sind durch eine entsprechende Reglerparametrierung im geschlossenen Regelkreis unbeobachtbar zu machen. Dies lässt sich entsprechend (15) durch $\boldsymbol{C} \boldsymbol{v}_{R_{k}}+\boldsymbol{D} \boldsymbol{p}_{k}=\mathbf{0}$ erreichen. Da darüber hinaus $\left(\lambda_{R_{k}} \boldsymbol{I}_{n}-\boldsymbol{A}\right) \boldsymbol{v}_{R_{k}}=\boldsymbol{B} \boldsymbol{p}_{k}$ gilt, lässt sich die Forderung

$\underbrace{\left[\begin{array}{cc}\lambda_{R_{k}} \boldsymbol{I}_{n}-\boldsymbol{A} & -\boldsymbol{B} \\ \boldsymbol{C} & \boldsymbol{D}\end{array}\right]}_{\boldsymbol{\Sigma}_{k}}\left[\begin{array}{c}\boldsymbol{v}_{R_{k}} \\ \boldsymbol{p}_{k}\end{array}\right]=\mathbf{0} \forall k=\delta+1, \ldots, n$

aufstellen. Darin ist $\boldsymbol{\Sigma}_{k} \in \mathbb{R}^{\left(n+n_{y}\right) \times\left(n+n_{u}\right)}$ die Rosenbrock'sche Systemmatrix. Da sie für $n_{u}=n_{y}$ quadratisch ist, hat (19) in einem solchen Fall nur dann eine nichttriviale Lösung, wenn $\boldsymbol{\Sigma}_{k}$ einen Rangabfall hat. Dies ist genau dann der Fall, wenn $\lambda_{R_{k}}$ einer Invarianten Nullstelle von $(\boldsymbol{A}, \boldsymbol{B}, \boldsymbol{C}, \boldsymbol{D})$ entspricht [32]. Aus diesem Grund können nicht-minimalphasige Systeme bei $n_{u}=n_{y}$ im Allgemeinen nicht statisch stabil entkoppelt werden. Für eine stabile Entkopplung sind dann dynamische Regler nötig $[6 ; 7 ; 17 ; 23])$. Eine Ausnahme bilden Systeme, bei denen die Invarianten Nullstellen sogenannte noninterconnecting-zeros sind, die als Nullstellen in den Hauptdiagonalelementen von $\boldsymbol{G}_{\boldsymbol{y} w}(s)$ auftreten $[14 ; 18]$.

Im Fall $n_{u}>n_{y}$ mit $\operatorname{rang}(\boldsymbol{B})=n_{u}$ folgt aus Lemma 1, dass der Kern von $\boldsymbol{\Sigma}_{k}$ die Dimension $n_{u}-n_{y}$ hat, solange die Rosenbrock'sche Systemmatrix an der Stelle $\lambda_{R_{k}}$ keinen Rangabfall gegenüber dem Normalrang aufweist. Demnach ist $\boldsymbol{\Sigma}_{k}^{\perp} \in \mathbb{R}^{\left(n+n_{u}\right) \times\left(n_{u}-n_{y}\right)}$ für fast alle $\lambda_{R_{k}}$. Somit 
genügen alle Rechtseigenvektoren und Parametervektoren, welche durch

$\left[\begin{array}{c}\boldsymbol{v}_{R_{k}} \\ \boldsymbol{p}_{k}\end{array}\right]=\boldsymbol{\Sigma}_{k}^{\perp} \boldsymbol{z}_{k}$

parametriert sind, der Bedingung (19). Dabei ist $\boldsymbol{z}_{k} \neq \mathbf{0} \in \mathbb{R}^{n_{u}-n_{y}}$ frei wählbar. Entscheidend ist, dass im Gegensatz zum quadratischen Fall Einfluss auf den im geschlossenen Regelkreis unbeobachtbaren Eigenwert $\lambda_{R_{k}}$ genommen werden werden kann. Dies liegt darin begründet, dass Ausgangsentkopplungsnullstellen in nicht-quadratischen Systemen nicht notwendigerweise auch Invariante Nullstellen sind (s. z. B. [29; 32]). Weist das System $(\boldsymbol{A}, \boldsymbol{B}, \boldsymbol{C}, \boldsymbol{D})$ Invariante Nullstellen auf, so ist (20) zwar bei beliebiger Wahl von $\lambda_{R_{k}}$ lösbar, allerdings ergeben sich in diesem Fall linear abhängige Rechtseigenvektoren $\boldsymbol{v}_{R_{k}}$. Werden die Invarianten Nullstellen jedoch kompensiert, d.h. wird $\lambda_{R_{k}}$ entsprechend einer Invarianten Nullstelle gewählt, so vergrößert sich die Dimension von $\boldsymbol{\Sigma}_{k}^{\perp}$ und es lassen sich linear unabhängige Rechtseigenvektoren finden. Diese Tatsache begründet Annahme 2, Invariante Nullstellen müssen also auch in nicht-quadratischen Systemen kompensiert werden. Außerdem ist dadurch erklärt, warum einige der unbeobachtbaren Regelungseigenwerte beim Entwurf gemäß Abschnitt 3.1 durch die Freiheitsgrade $\boldsymbol{R}_{K}$ verändert werden und einige nicht.

Im Fall $n_{y}<\operatorname{rang}(\boldsymbol{B})<n_{u}$ verringert sich der Normalrang von $\boldsymbol{\Sigma}_{k}$ und damit auch die Dimension von $\boldsymbol{\Sigma}_{k}^{\perp}$. Somit stehen weniger Freiheitsgrade bezüglich $\boldsymbol{v}_{R_{k}}$ und $\boldsymbol{p}_{k}$ zur Verfügung.

Nachdem über (16), (18) und (20) alle Parameterund Rechtseigenvektoren bestimmt sind, ergibt sich mit $\boldsymbol{P}=\left[\begin{array}{lll}\boldsymbol{p}_{1} & \ldots & \boldsymbol{p}_{n}\end{array}\right]$ die Reglermatrix zu

$\boldsymbol{K}_{p}=-\boldsymbol{P} \boldsymbol{V}_{R}^{-1}$.

Sie stellt sicher, dass die Eigenwerte von $\boldsymbol{A}-\boldsymbol{B} \boldsymbol{K}$ entweder bestimmten Zeilen in $\boldsymbol{G}_{\boldsymbol{y} w}(s)$ als Pole zugewiesen werden (18) oder aber im geschlossenen Regelkreis unbeobachtbar gemacht werden (20).

Aus der Forderung der spezifizierten stationären Verstärkung ergibt sich für das Vorfilter die Bestimmungsgleichung

$\underbrace{\left(-\left(\boldsymbol{C}-\boldsymbol{D} \boldsymbol{K}_{p}\right)\left(\boldsymbol{A}-\boldsymbol{B} \boldsymbol{K}_{p}\right)^{-1} \boldsymbol{B}+\boldsymbol{D}\right)}_{\boldsymbol{\Lambda}} \boldsymbol{F}_{p}=S$

mit $S=\operatorname{diag}\left(z_{1,0} / q_{1,0}, \ldots, z_{n_{y}, 0} / q_{n_{y}, 0}\right)$. Daraus folgt schließlich

$\boldsymbol{F}_{p}=\boldsymbol{\Lambda}^{+} \boldsymbol{S}+\boldsymbol{\Lambda}^{\perp} \boldsymbol{Z}$

mit einer frei wählbaren Matrix $\boldsymbol{Z} \in \mathbb{R}^{\left(n_{u}-n_{y}\right) \times n_{y}}$. Das Vorfilter (23) gewährleistet für die Nichtdiagonalemente von $\boldsymbol{G}_{\boldsymbol{y} w}(s)$ zunächst $g_{i, j}(s=0)=0$. Es impliziert jedoch darüber hinaus, dass $g_{i, j}(s)=0 \forall s \in \mathbb{C}$ gilt. Denn andernfalls müsste ein Nichtdiagonalelement $g_{i, j}(s)$ eine Nullstelle aufweisen. Dies führt jedoch auf einen Widerspruch, da allen Elementen der $i$-ten Zeile von $\boldsymbol{G}_{\boldsymbol{y} w}(s)$ durch (21) $\delta_{i}$ Pole zugewiesen wurden und die Differenzenordnungen nach [9] invariant bezüglich Zustandsrückführungen sind. Analog zur Argumentation in [20, Abschnitt 2.2.3] ergibt sich also, dass (23) in Verbindung mit (21) nicht nur im stationären Fall Entkopplung sicherstellt, sondern für alle $s \in \mathbb{C}$. Somit ist (3) erreicht.

Der parametrische Entwurf erlaubt im Vergleich $\mathrm{zu}$ Abschnitt 3.1 einen tieferen Einblick in die Bedeutung der entstehenden zusätzlichen Freiheitsgrade. Neben der Vorgabe der Richtung der Parametervektoren im von $\Pi_{i j}^{\perp}$ aufgespannten Unterraum können auch die unbeobachtbaren Regelungseigenwerte $\lambda_{R_{k}}$ gezielt vorgegeben werden, sofern sie nicht zur Kompensation Invarianter Nullstellen herangezogen werden müssen. Weiterhin können die zugehörigen Parameter- und Rechtseigenvektoren beeinflusst werden.

Anmerkung 1. In Satz 1 kann anstatt $\left(\boldsymbol{F}_{0}, \boldsymbol{K}_{0}\right)$ auch eine mit Hilfe des parametrischen Entwurfs generierte Reglerparametrierung $\left(\boldsymbol{F}_{p}, \boldsymbol{K}_{p}\right)$ verwendet werden. Da $\left(\boldsymbol{F}_{p}, \boldsymbol{K}_{p}\right)$ Führungsentkopplung gewährleistet, gilt $D^{*} F_{p}=N$ und $\boldsymbol{D}^{*} \boldsymbol{K}_{p}=\boldsymbol{M}$. Da auch $\left(\boldsymbol{F}_{0}, \boldsymbol{K}_{0}\right)$ die Entkopplungsbedingung (12) erfüllt, gilt $\boldsymbol{D}^{*}\left(\boldsymbol{K}_{p}-\boldsymbol{K}_{0}\right)=\mathbf{0}$. Die Differenz $\boldsymbol{K}_{p}-\boldsymbol{K}_{0}$ liegt also ebenso im Nullraum von $\boldsymbol{D}^{*}$ wie $\boldsymbol{F}_{p}-\boldsymbol{F}_{0}$ und $D^{* \perp}$ stellt gerade eine Basis dieses Nullraumes dar.

\section{Nutzung der zusätzlichen Freiheitsgrade}

Die in Abschnitt 3 herausgearbeiteten zusätzlichen Freiheitsgrade gegenüber quadratischen Entkopplungsregelungen werden im Folgenden für zwei verschiedene Anwendungen gezielt genutzt.

\subsection{Minimierung der Stellenergie}

Zunächst wird ein Verfahren vorgestellt, welches die gezielte Ausnutzung der zusätzlichen Freiheitsgrade $\boldsymbol{R}_{K}$ und $\boldsymbol{R}_{F}$ zur Senkung der Stellenergie ermöglicht.

Um die auftretenden Stellgrößen bei einer Führungsregelung $\mathrm{zu}$ untersuchen, wird die Übertragungsfunktion $\boldsymbol{G}_{\boldsymbol{u} \boldsymbol{w}}(s)$ betrachtet, welche die Wirkung der Führungsgrößen auf die Stellgrößen beschreibt. Anhand von (1) und (2) ergibt sich die Zustandsraumdarstellung

$\dot{\boldsymbol{x}}=(\boldsymbol{A}-\boldsymbol{B K}) \boldsymbol{x}+\boldsymbol{B F} \boldsymbol{w}$,

$\boldsymbol{u}=-\boldsymbol{K} \boldsymbol{x}+\boldsymbol{F} \boldsymbol{w}$.

Es lässt sich zeigen [3], dass die Beschränkung der Energieverstärkung $\gamma^{2}$ des Systems (24), welche sich aus

$\int_{0}^{\infty} \boldsymbol{u}^{\top}(t) \cdot \boldsymbol{u}(t) d t<\gamma^{2} \int_{0}^{\infty} \boldsymbol{w}^{\top}(t) \cdot \boldsymbol{w}(t) d t$

ergibt, gleichbedeutend ist mit $\left\|\boldsymbol{G}_{\boldsymbol{u} \boldsymbol{w}}(s)\right\|_{\infty}<\gamma$. Um also eine möglichst geringe Energieverstärkung zwischen Führungsgrößen und Stellgrößen $\mathrm{zu}$ erreichen, wird eine Minimierung von $\left\|\boldsymbol{G}_{\boldsymbol{u} \boldsymbol{w}}(s)\right\|_{\infty}$ angestrebt. Nach Lemma 2 


$$
\begin{array}{r}
{\left[\begin{array}{ccc}
\operatorname{He}\left(\left(\boldsymbol{A}-\boldsymbol{B} \boldsymbol{K}_{0}\right) \boldsymbol{Q}-\boldsymbol{B} \boldsymbol{D}^{* \perp} \boldsymbol{Y}\right) & \boldsymbol{B}\left(\boldsymbol{F}_{0}+\boldsymbol{D}^{* \perp} \boldsymbol{R}_{F}\right) & -\left(\boldsymbol{K}_{0} \boldsymbol{Q}+\boldsymbol{D}^{* \perp} \boldsymbol{Y}\right)^{\top} \\
* & -\gamma \boldsymbol{I}_{n_{y}} & \left(\boldsymbol{F}_{0}+\boldsymbol{D}^{* \perp} \boldsymbol{R}_{F}\right)^{\top} \\
* & * & -\gamma \boldsymbol{I}_{n_{u}}
\end{array}\right] \prec \mathbf{0}} \\
\boldsymbol{\Theta}_{i}=\left[\begin{array}{ccc}
\operatorname{He}\left(\left(\boldsymbol{A}-\boldsymbol{B} \boldsymbol{K}_{0}\right) \boldsymbol{Q}-\boldsymbol{B} \boldsymbol{D}^{* \perp} \boldsymbol{Y}\right) & \boldsymbol{B}\left(\boldsymbol{F}_{0}+\boldsymbol{D}^{* \perp} \boldsymbol{R}_{F}\right) & -\left(\boldsymbol{e}_{i}^{\top}\left(\boldsymbol{K}_{0} \boldsymbol{Q}+\boldsymbol{D}^{* \perp} \boldsymbol{Y}\right)\right)^{\top} \\
* & -\gamma_{i} \boldsymbol{I}_{n_{y}} & \left(\boldsymbol{e}_{i}^{\top}\left(\boldsymbol{F}_{0}+\boldsymbol{D}^{* \perp} \boldsymbol{R}_{F}\right)\right)^{\top} \\
* & * & -\gamma_{i}
\end{array}\right]
\end{array}
$$

lässt sich dies als ein Optimierungsproblem mit Matrixungleichungen als Nebenbedingungen beschreiben, und zwar

$\underset{X, R_{K}, R_{F}}{\operatorname{minimiere}} \gamma$ u.d. B. d.

$\boldsymbol{X} \succ \mathbf{0}$,

$\left[\begin{array}{ccc}\operatorname{He}(\boldsymbol{X}(\boldsymbol{A}-\boldsymbol{B} \boldsymbol{K})) & \boldsymbol{X}(\boldsymbol{B} \boldsymbol{F}) & -\boldsymbol{K}^{\top} \\ * & -\gamma \boldsymbol{I}_{n_{y}} & \boldsymbol{F}^{\top} \\ * & * & -\gamma \boldsymbol{I}_{n_{u}}\end{array}\right] \prec \mathbf{0}$

Dabei handelt es sich bei (26c) um eine bilineare Matrixungleichung (BMI), da wegen $K=\boldsymbol{K}_{0}+\boldsymbol{D}^{* \perp} \boldsymbol{R}_{K}$ Produktterme der Form $\boldsymbol{X D}^{* \perp} \boldsymbol{R}_{K}$ in den Variablen auftreten. Diese lassen sich jedoch eliminieren, indem die Matrix $Q=X^{-1}$ eingeführt wird und (26c) beidseitig mit der positiv definiten, symmetrischen Blockdiagonalmatrix $\boldsymbol{\Gamma}=\operatorname{diag}\left(\boldsymbol{Q}, \boldsymbol{I}_{n_{y}}, \boldsymbol{I}_{n_{u}}\right)$ multipliziert wird. Mittels dieser Kongruenztransformation ergibt sich eine äquivalente Beschreibung des Problems mit den Nebenbedingungen $\boldsymbol{Q} \succ \mathbf{0}$ und

$\left[\begin{array}{ccc}\operatorname{He}((\boldsymbol{A}-\boldsymbol{B} \boldsymbol{K}) \boldsymbol{Q}) & \boldsymbol{B} \boldsymbol{F} & -(\boldsymbol{K} \boldsymbol{Q})^{\top} \\ * & -\gamma \boldsymbol{I}_{n_{y}} & \boldsymbol{F}^{\top} \\ * & * & -\gamma \boldsymbol{I}_{n_{u}}\end{array}\right] \prec \mathbf{0}$.

Die nun noch vorhandenen Produktterme in den Variablen lassen sich durch die Substitution $R_{K} Q=Y \in$ $\mathbb{R}^{\left(n_{u}-n_{y}\right) \times n}$ eliminieren. Es ergibt sich somit das LMIProblem

$\underset{Q Y R_{\mp}}{\operatorname{minimiere}} \gamma$ u.d.B.d. $\mathbf{Q} \succ \mathbf{0},(29)$,

wobei (29) oben auf dieser Seite angegeben ist. Die gesuchte Matrix $\boldsymbol{R}_{K}$ erhält man dann nach Lösung des Optimierungsproblems mittels der Rücktransformation $\boldsymbol{R}_{K}=Y_{Q^{-1}}$.

Anmerkung 2. Nach [2, Proposition 8.2.7] impliziert (26c), dass $\mathrm{He}(\boldsymbol{X}(\boldsymbol{A}-\boldsymbol{B} \boldsymbol{K})) \prec \mathbf{0}$ gilt. Folglich wird durch (26c) bzw. (29) neben der Beschränkung der Energieverstärkung auch die Stabilität des geschlossenen Regelkreises sichergestellt.

Das Verfahren lässt sich in einfacher Art und Weise erweitern, wenn nicht die generelle Energieverstärkung zwischen Führungs- und Stellgrößen minimiert werden soll, sondern einzelne Stellgrößen gezielt zu beeinflussen sind. Dies kann z. B. dadurch motiviert sein, dass für die verschiedenen Aktoren unterschiedliche Belastungsgrenzen gelten oder indirekt Stellgrößenbeschränkungen eingehalten werden sollen. In diesem Fall wird für jede Stellgröße eine individuelle Schranke $\gamma_{i}$ eingeführt, welche die maximale Energieverstärkung von $\boldsymbol{w}$ auf $u_{i}$ beschreibt. Mittels frei wählbarer Gewichtungskoeffizienten $\alpha_{i} \geq 0$ erhält man das Optimierungsproblem

$\underset{Q, Y, R_{F}}{\operatorname{minimiere}} \sum_{i=1}^{n_{u}} \alpha_{i} \gamma_{i}$ u.d. B.d.

$Q \succ 0$,

$\boldsymbol{\Theta}_{i} \prec \mathbf{0}, \forall i=1, \ldots, n_{u}$.

Dabei sind die Matrizen $\boldsymbol{\Theta}_{i}$ in (31) oben auf dieser Seite angegeben. Darin bezeichnet $\boldsymbol{e}_{i}$ einen Einheitsvektor mit dem Element 1 in der $i$-ten Zeile.

Anmerkung 3. Da es sich bei (28) und (30) um konvexe Optimierungsprobleme handelt, hat die Wahl von $\left(\boldsymbol{F}_{0}, \boldsymbol{K}_{0}\right)$ bzw. $\left(\boldsymbol{F}_{p}, \boldsymbol{K}_{p}\right)$ keinen Einfluss auf die sich ergebende Lösung $\boldsymbol{K}_{\mathrm{opt}}, \boldsymbol{F}_{\text {opt }}$. Sie wird lediglich durch unterschiedliche Werte für $\boldsymbol{R}_{K}$ und $\boldsymbol{R}_{F}$ erzeugt.

Anmerkung 4. Die Regelungseigenwerte des Systems können mittels geeigneter LMIs auf bestimmte Bereiche beschränkt werden [5]. Um beispielsweise eine ausreichende Abklingrate $\zeta>0$ bezüglich eventueller Anfangsauslenkungen zu gewährleisten, wird

$\mathrm{He}\left(\left(\boldsymbol{A}-\boldsymbol{B} \boldsymbol{K}_{0}\right) \boldsymbol{Q}-\boldsymbol{B D}^{* \perp} \boldsymbol{Y}\right)+2 \zeta \boldsymbol{Q} \prec \mathbf{0}$

als zusätzliche Nebenbedingung in (28) bzw. (30) hinzugefügt.

\subsection{Optimierung der Robustheit}

Bisher wurde vorausgesetzt, dass ein exaktes Modell der Regelstrecke existiert. Dies ist in der Praxis jedoch nie der Fall. Durch Ungenauigkeiten in der Modellierung, Alterungseffekte oder auch durch Linearisierungsfehler treten parametrische Unsicherheiten auf, die im Folgenden durch die Beschreibung

$\dot{x}=A_{\xi} x+B_{\xi} u$,

$y=C_{\xi} x+D_{\xi} u$

erfasst werden. Dabei gilt

$\left[\begin{array}{cc}A_{\xi} & B_{\xi} \\ C_{\xi} & D_{\xi}\end{array}\right] \in \Omega$,

$\Omega=\left\{\left[\begin{array}{ll}\boldsymbol{A}_{\boldsymbol{\xi}} & \boldsymbol{B}_{\xi} \\ \boldsymbol{C}_{\boldsymbol{\xi}} & \boldsymbol{D}_{\xi}\end{array}\right]=\sum_{i=1}^{N} \xi_{i}\left[\begin{array}{cc}\boldsymbol{A}_{i} & \boldsymbol{B}_{i} \\ \boldsymbol{C}_{i} & \boldsymbol{D}_{i}\end{array}\right], \sum_{i=1}^{N} \xi_{i}=1, \xi_{i} \geq 0\right\}$. 
Die Matrizen $\boldsymbol{A}_{\boldsymbol{\xi}}, \boldsymbol{B}_{\boldsymbol{\xi}}, \boldsymbol{C}_{\boldsymbol{\xi}}$ und $\boldsymbol{D}_{\boldsymbol{\xi}}$ lassen sich also als konvexe Kombinationen von Eckpunkten $i$ eines Polytops darstellen. Dabei wird vorausgesetzt, dass die Matrizen des nominalen Modells (1) durch (33) beschrieben werden können.

Wird nun ein Entkopplungsregler für das nominale System (1) entworfen, so führt dies im Allgemeinen dazu, dass das mit diesem Regler beeinflusste System (33) nicht mehr entkoppelt ist. Neben einer Verschiebung der vorgegebenen Pole treten insbesondere auch unerwünschte Verkopplungen auf. Ziel dieses Abschnittes ist es, die zusätzlichen Freiheitsgrade beim Entwurf von Entkopplungsreglern für nicht-quadratische Systeme zur Steigerung der Robustheit bezüglich der genannten parametrischen Unsicherheiten zu nutzen.

Dazu wird ein approximativer Model-Matching Ansatz herangezogen, wie er z. B. in [36] für eine robuste Fehlerdetektion eingesetzt wird. Grundidee ist es, die maximale Abweichung zwischen dem Übertragungsverhalten des geschlossenen Regelkreises für alle möglichen parametrischen Unsicherheiten und einem gegebenen Referenzmodell über alle Frequenzen $\mathrm{zu}$ minimieren. Als Referenzmodell $G_{\text {ref }}(s)$ wird dabei eine Minimalrealisierung des gewünschten entkoppelten Übertragungsverhaltens im nominalen Fall angesetzt. Da die einzelnen Übertragungspfade entkoppelt sind, bietet sich die Zustandsraumrealisierung

$$
\begin{aligned}
& \dot{\boldsymbol{x}}_{\mathrm{ref}}=\boldsymbol{A}_{\mathrm{ref}} \boldsymbol{x}_{\mathrm{ref}}+\boldsymbol{B}_{\mathrm{ref}} \boldsymbol{w}, \\
& y_{\text {ref }}=C_{\text {ref }} x_{\text {ref }}+D_{\text {ref }} \boldsymbol{w} \text {, } \\
& \boldsymbol{A}_{\text {ref }}=\operatorname{diag}\left(\boldsymbol{A}_{\mathrm{ref} 1}, \ldots, \boldsymbol{A}_{\mathrm{ref} n_{y}}\right) \text {, } \\
& \boldsymbol{B}_{\mathrm{ref}}=\operatorname{diag}\left(\boldsymbol{B}_{\mathrm{ref} 1}, \ldots, \boldsymbol{B}_{\mathrm{ref}_{n_{y}}}\right) \text {, } \\
& C_{\text {ref }}=\operatorname{diag}\left(C_{\text {ref } 1}, \ldots, C_{\text {ref } n_{y}}\right) \text {, } \\
& \boldsymbol{D}_{\text {ref }}=\operatorname{diag}\left(\boldsymbol{D}_{\text {ref } 1}, \ldots, \boldsymbol{D}_{\text {ref }_{n_{y}}}\right)
\end{aligned}
$$

an. Für Systeme ohne Durchgriff können die Matrizen $\left(\boldsymbol{A}_{\text {ref }}, \boldsymbol{B}_{\text {ref }_{i}}, C_{\text {ref }_{i}}\right)$ beispielsweise als Steuerungsnormalform der gewünschten Übertragungsfunktion $g_{i, i}(s)$ entsprechend (4) angenommen werden. Es gilt folglich $\boldsymbol{x}_{\mathrm{ref}} \in \mathbb{R}^{\delta}$ und in Zustandsraumdarstellung lässt sich $\boldsymbol{G}_{\boldsymbol{y} \boldsymbol{w}, i}(s)-\boldsymbol{G}_{\mathrm{ref}}(s)$ mit $\overline{\boldsymbol{x}}_{i}=\left[\boldsymbol{x}_{i}^{\top} \boldsymbol{x}_{\mathrm{ref}}^{\top}\right]^{\top} \in \mathbb{R}^{n+\delta}$ beschreiben als

$\dot{\overline{\boldsymbol{x}}}_{i}=\underbrace{\left[\begin{array}{cc}\boldsymbol{A}_{i}-\boldsymbol{B}_{i} \boldsymbol{K} & \mathbf{0} \\ \mathbf{0} & \boldsymbol{A}_{\mathrm{ref}}\end{array}\right]}_{\boldsymbol{A}_{\boldsymbol{z} w, i}} \overline{\boldsymbol{x}}_{i}+\underbrace{\left[\begin{array}{c}\boldsymbol{B}_{i} \boldsymbol{F} \\ \boldsymbol{B}_{\mathrm{ref}}\end{array}\right]}_{\boldsymbol{B}_{\boldsymbol{z} w, i}} \boldsymbol{w}$,

$$
z=\underbrace{\left[\begin{array}{ll}
C_{i}-D_{i} K & -C_{\mathrm{ref}}
\end{array}\right]}_{C_{z w, i}} \bar{x}_{i}+\underbrace{\left(D_{i}-D_{\mathrm{ref}}\right)}_{D_{z w, i}} w .
$$

Der Performance-Ausgang $\boldsymbol{z}=\boldsymbol{y}-\boldsymbol{y}_{\text {ref }} \in \mathbb{R}^{n_{y}}$ beschreibt dabei die Abweichung der Ausgänge von geregeltem System und Referenzmodell. Die Minimierung der maximalen Abweichung von $\boldsymbol{G}_{\boldsymbol{y} w, i}(s)$ zum Referenzmo- dell $\boldsymbol{G}_{\text {ref }}(s)$ lässt sich daher mit $\boldsymbol{G}_{\boldsymbol{z}, i}(s)=\boldsymbol{C}_{\boldsymbol{z} \boldsymbol{w}, i}\left(s \boldsymbol{I}_{n+\delta}-\right.$ $\left.\boldsymbol{A}_{\boldsymbol{z} w, i}\right)^{-1} \boldsymbol{B}_{\boldsymbol{z} w, i}+\boldsymbol{D}_{z \boldsymbol{w}, i}$ als Optimierungsproblem

$\underset{R_{K}, R_{f}}{\operatorname{minimiere}} \beta$ u.d. B.d.

$\max _{i=1, \ldots, N}\left\|\boldsymbol{G}_{z w, i}(s)\right\|_{\infty}<\beta$

formulieren.

Mit Hilfe von Lemma 2 lässt sich (37b) unter Verwendung von (36) in die Matrixungleichungen

$\boldsymbol{X} \succ \mathbf{0}$,

$\left[\begin{array}{ccc}\operatorname{He}\left(\boldsymbol{X}_{\boldsymbol{z} \boldsymbol{w}, i}\right) & \boldsymbol{X B}_{\boldsymbol{z} \boldsymbol{w}, i} & \boldsymbol{C}_{\boldsymbol{z}, i}^{\top} \\ * & -\beta \boldsymbol{I}_{n_{y}} & \boldsymbol{D}_{\boldsymbol{z w}, i}^{\top} \\ * & * & -\boldsymbol{\beta} \boldsymbol{I}_{n_{y}}\end{array}\right] \prec \mathbf{0}, i=1, \ldots, N$

überführen. Zu beachten ist dabei, dass in (38) aufgrund der gemeinsamen Lyapunov-Matrix $\boldsymbol{X} \in \mathbb{R}^{(n+\delta) \times(n+\delta)}$ lediglich die endliche Anzahl $N$ von Nebenbedingungen zu berücksichtigen ist, um $\beta$ zu bestimmen. Diese Schranke gilt nach [3] auch für alle Systeme innerhalb der konvexen Hülle der Eckpunkte (34).

Führt man nun $\boldsymbol{Q}=\boldsymbol{X}^{-1}$ ein und multipliziert (38b) von beiden Seiten mit der positiv definiten Matrix $\boldsymbol{\Gamma}=$ $\operatorname{diag}\left(\boldsymbol{Q}, \boldsymbol{I}_{n_{y}}, \boldsymbol{I}_{n_{y}}\right)$, so lässt sich für (38)

$Q \succ \mathbf{0}$,

$\left[\begin{array}{ccc}\operatorname{He}\left(\boldsymbol{A}_{\boldsymbol{z}, i} \boldsymbol{Q}\right) & \boldsymbol{B}_{\boldsymbol{z} \boldsymbol{w}, i} & \boldsymbol{Q} \boldsymbol{C}_{\boldsymbol{z} \boldsymbol{w}, i}^{\top} \\ * & -\beta \boldsymbol{I}_{n_{y}} & \boldsymbol{D}_{\boldsymbol{z} \boldsymbol{w}, i}^{\top} \\ * & * & -\beta \boldsymbol{I}_{n_{y}}\end{array}\right] \prec \mathbf{0}, i=1, \ldots, N$

schreiben. Bei (39b) handelt es sich jedoch ebenso wie bei (38b) um eine BMI, da Produktterme in den Variablen $\boldsymbol{Q}$ und $\boldsymbol{R}_{K}$ auftreten. Diese lassen sich im Gegensatz zu Abschnitt 4.1 nicht in einfacher Weise durch eine Variablensubstitution eliminieren. Partitioniert man

$\boldsymbol{Q}=\left[\begin{array}{ll}\boldsymbol{Q}_{11} & \boldsymbol{Q}_{12} \\ \boldsymbol{Q}_{12}^{\top} & \mathbf{Q}_{22}\end{array}\right]$,

so wird deutlich, dass sowohl Terme $\boldsymbol{R}_{K} \boldsymbol{Q}_{11}$ als auch $\boldsymbol{R}_{K} \boldsymbol{Q}_{12}$ auftreten. Es ist nun möglich, eine blockdiagonale Lyapunovmatrix anzusetzen, also $\boldsymbol{Q}_{12}=\mathbf{0} \mathrm{zu}$ fordern. Damit kann das Problem wie in Abschnitt 4.1 in ein konvexes Optimierungsproblem überführt werden. Dieser Ansatz, der z. B. in anderem Zusammenhang in [36] verfolgt wird, führt aufgrund der strukturellen Beschränkung von $\boldsymbol{Q}$ jedoch zu sehr konservativen Ergebnissen. Aus diesem Grund wird hier ein alternatives Verfahren zur Lösung des BMI-Problems (37) vorgeschlagen, welches ebenso wie z.B. der Path-Following Algorithmus [26] ausgehend von einer initialen Lösung iterativ arbeitet.

Dabei wird zunächst für eine initiale Lösung $\left(\boldsymbol{F}_{0}, \boldsymbol{K}_{0}\right)$ bzw. $\left(\boldsymbol{F}_{p}, \boldsymbol{K}_{p}\right)$ (s. Anmerkung 1) mit $\boldsymbol{R}_{K}=\mathbf{0}$ und $\boldsymbol{R}_{F}=\mathbf{0}$ eine initiale Schranke $\beta$ sowie eine zugehörige Lyapu- 
novmatrix $\boldsymbol{Q}$ ermittelt. Dies ist ohne Weiteres möglich, da in diesem Fall ein LMI-Problem vorliegt. Im Folgenden wird das Problem (37) bzw. (39) iterativ gelöst. Jeder Schleifendurchlauf besteht dabei aus zwei Schritten. Zunächst ist das jeweils aktuelle $Q$ fix, also keine LMI-Variable. Die Schranke $\beta$ wird anhand der Matrizen $\boldsymbol{R}_{K}$ und $\boldsymbol{R}_{F}$ minimiert. Im zweiten Schritt ist das zuvor gefundene $\boldsymbol{R}_{K}$ fix und $\beta$ wird mittels $Q$ und $\boldsymbol{R}_{F}$ minimiert. In beiden Teilschritten liegen also jeweils LMI-Probleme vor. Das Verfahren wird terminiert, nachdem ein geeignetes Abbruchkriterium erfüllt ist. Dies kann z.B. eine feste Anzahl von Iterationen sein oder das Unterschreiten einer Schranke für die in der jeweiligen Iteration erzielten Verbesserung von $\beta$.

\section{Algorithmus 1.}

1. Ermittle $\left(\boldsymbol{F}_{0}, \boldsymbol{K}_{0}\right)$ entsprechend Satz 1 bzw. $\left(\boldsymbol{F}_{p}, \boldsymbol{K}_{p}\right)$ entsprechend Abschnitt 3.2 (s. Anmerkung 1). Setze $\boldsymbol{R}_{K}=\mathbf{0}$, $\boldsymbol{R}_{F}=\mathbf{0}$ und löse LMI-Problem

$\underset{\mathbf{Q}}{\operatorname{minimiere}} \beta$ u.d.B.d. $\mathbf{Q} \succ \mathbf{0},(39 \mathrm{~b})$

Nenne gefundene Lyapunovmatrix $\boldsymbol{Q}_{f}$.

2. Löse LMI-Problem

$\underset{\boldsymbol{R}_{K}, \boldsymbol{R}_{F}}{\operatorname{minimiere}} \beta$ u.d.B.d. (39b), $\boldsymbol{Q}=\boldsymbol{Q}_{f}$,

wobei $Q_{f}$ fix ist. Nenne gefundene Matrix des Reglers $\boldsymbol{R}_{K, f}$.

3. Löse LMI-Problem

$\underset{Q}{\operatorname{minimiere}} \beta$ u.d.B.d. $\boldsymbol{Q} \succ \mathbf{0}, \boldsymbol{R}_{K}=\boldsymbol{R}_{K, f},(39 \mathrm{~b})$,

wobei $\boldsymbol{R}_{K, f}$ fix ist. Nenne gefundene Lyapunovmatrix $\boldsymbol{Q}_{f}$. Falls Abbruchkriterium erfüllt, terminiere Algorithmus. Andernfalls gehe zu 2.

Da es sich bei (37) um ein BMI-Problem handelt, kann nicht garantiert werden, dass Algorithmus 1 konvergiert. Außerdem wird das Verfahren im Allgemeinen lediglich ein lokales Optimum liefern. Nach Satz 1 sind jedoch mit $\boldsymbol{K}_{0}=\boldsymbol{D}^{*+} \boldsymbol{M}, \boldsymbol{F}_{0}=\boldsymbol{D}^{*+} \boldsymbol{N}$ und dem parametrischen Entwurf aus Abschnitt 3.2 gültige initiale Lösungen bekannt. Die durch Algorithmus 1 generierte Lösung ist dann im Sinne des Optimierungskriteriums (37a) immer besser oder mindestens ebenso gut wie die initiale Lösung.

Alternativ zum diskutierten Vorgehen ist es auch möglich, die in Abschnitt 3.2 herausgearbeiteten Freiheitsgrade der Vollständigen Modalen Synthese für die Optimierung der Robustheit zu nutzen. Ein verwandtes Vorgehen wurde in [13] vorgestellt. Die im vorliegenden Beitrag beschriebene Optimierung zeichnet sich jedoch gegenüber [13] durch eine wesentlich geringere Anzahl einzustellender Parameter aus. Es ist lediglich ein Startwert zu wählen, was die Anwendung vereinfacht.

Anmerkung 5. Selbstverständlich lassen sich die beiden Optimierungsprobleme (37) und (28) bzw. (30) aus diesem und dem Abschnitt 4.1 kombinieren, indem ein gewichteter Mittelwert der Gütemaße als Optimierungskriterium gewählt wird. Beim Entwurf des Entkopplungsreglers kann dann eine Abwägung zwischen geringer Energieverstärkung bezüglich der Stellgrößen und guter Robustheit bezüglich unsicherer Modellparameter getroffen werden.

Anmerkung 6. Wird lediglich (37b) als Nebenbedingung betrachtet, so kann dies in Algorithmus $1 \mathrm{zu}$ einem betragsmäßig unbeschränkten Anwachsen unbeobachtbarer Eigenwerte führen. Aus diesem Grund ist es sinnvoll, die Regelungseigenwerte - und damit auch die unbeobachtbaren Eigenwerte - ähnlich wie in Anmerkung 4 auf bestimmte Eigenwertbereiche zu beschränken. Um eine ausreichende Dämpfung zu gewährleisten und gleichzeitig den Betrag der Eigenwerte zu beschränken kann z. B. ein kreisförmiges Gebiet um den Ursprung mit Radius $\varrho$ vorgegeben werden. Mit $\mathbb{R}^{n \times n} \ni \boldsymbol{Q}_{\mathrm{ew}} \succ \mathbf{0}$ werden dazu die Nebenbedingungen

$\left[\begin{array}{cc}-\varrho \boldsymbol{Q}_{\mathrm{ew}} & \left(\boldsymbol{A}_{i}-\boldsymbol{B}_{i}\left(\boldsymbol{K}_{0}+\boldsymbol{D}^{* \perp} \boldsymbol{R}_{K}\right)\right) \boldsymbol{Q}_{\mathrm{ew}} \\ * & -\varrho \boldsymbol{Q}_{\mathrm{ew}}\end{array}\right] \prec \mathbf{0}$

in die Optimierungsprobleme aus Algorithmus 1 eingebunden.

\section{Beispiel}

Die Ergebnisse sollen beispielhaft anhand der linearisierten Longitudinaldynamik einer F18-HARV (High Angle of Attack Research Vehicle) aus [33] verdeutlicht werden. Der Zustandsvektor $\boldsymbol{x}=\left[\begin{array}{lll}v & \alpha & q\end{array}\right]^{\top}$ setzt sich aus der Longitudinalgeschwindigkeit, dem Anstellwinkel sowie Nickrate und -winkel zusammen. Die $n_{u}=6$ Eingangsgrößen sind Schubvektorrichtung, symmetrischer Querruderausschlag, Stabilatorwinkel, Krügerklappenwinkel, Landeklappenwinkel und Schubstärke. Dabei sind sowohl Zustands- als auch Eingangsgrößen als Abweichungen zum Arbeitspunkt bei einer Longitudinalgeschwindigkeit von $262,25 \mathrm{~km} / \mathrm{h}$, einem Nickwinkel von $0^{\circ}$ und einem Anstellwinkel von $25^{\circ} \mathrm{zu}$ verstehen. Es ergeben sich somit

$A=\left[\begin{array}{cccc}-0,075 & -24,05 & 0 & -32,16 \\ -0,0009 & -0,196 & 0,9896 & 0 \\ -0,0002 & -0,1454 & -0,1677 & 0 \\ 0 & 0 & 1 & 0\end{array}\right]$,

$\boldsymbol{B}=\left[\begin{array}{cccc}-1,15 & -0,01 & -0,335 & 0 \\ 0 & -0,005 & -0,035 & 0 \\ -2,482 & -0,0136 & -0,408 & 0 \\ 0,0786 & 0 & -0,0012 & 0 \\ -2,466 & -0,018 & -0,042 & 0 \\ 4,32 & -0,008 & 0,0135 & 0\end{array}\right]^{\top}$. 

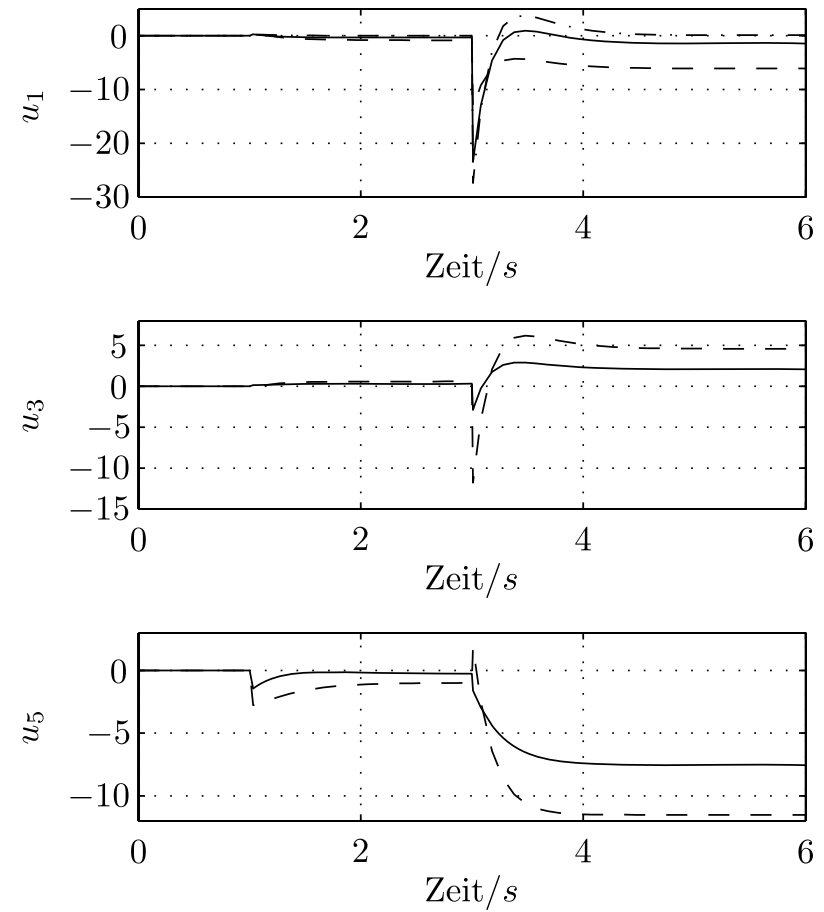
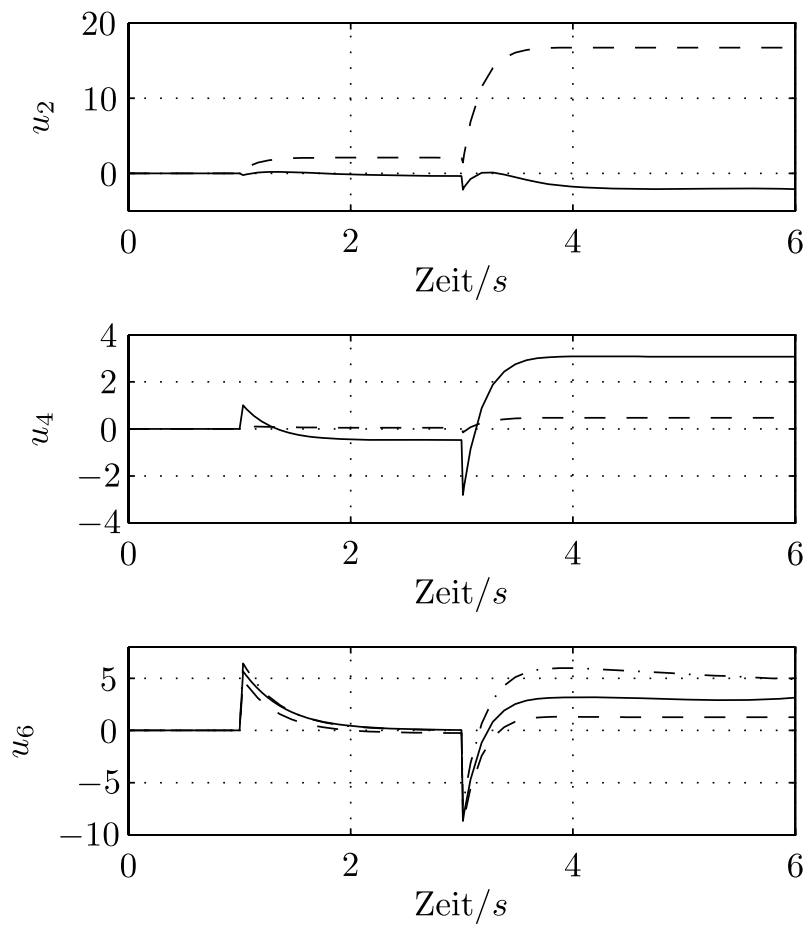

Bild 1 Stellgrößenverlauf im quadratischen (-·), überaktuierten (--) und optimierten überaktuierten (-) System.

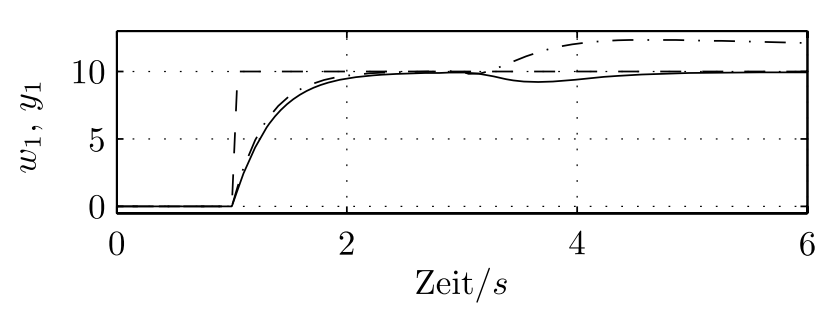

Bild 2 Verkopplung bei quadratischem (---) und optimiertem überaktuiertem (-) System.

Geregelt werden die Longitudinalgeschwindigkeit $y_{1}=x_{1}$ und der Anstellwinkel $y_{2}=x_{4}$. Zu Vergleichszwecken wird ein Entkopplungsregler für das quadratische System mit den Eingängen Schubvektorwinkel und Schubstärke entworfen. Die Differenzenordnungen sind nach (5) sowohl im quadratischen als auch im überaktuierten Fall $\delta_{1}=1$ und $\delta_{2}=2$. Als Pole der Übertragungsmatrix $G_{y w}(s)$ werden $\lambda_{1,1}=-3, \lambda_{2,1}=-4$ und $\lambda_{2,2}=-5$ vorgeben. Da das quadratische System über eine Invariante Nullstelle bei $\eta=-0,2380$ verfügt, werden Störungen und Anfangsauslenkungen nur sehr langsam ausgeregelt. Mittels der parametrischen Methode aus Abschnitt 3.2 wird ein Regler für das überaktuierte System entworfen, welcher den unbeobachtbaren Eigenwert bei $\lambda_{4}=-6$ platziert. Dies ist möglich, da das nicht-quadratische System keine Invarianten Nullstellen aufweist und somit der unbeobachtbare Regelungseigenwert frei gewählt werden kann. Für die übrigen zusätzlichen Freiheitsgrade gilt

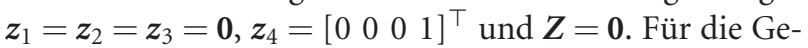
wichtung der einzelnen Aktoren wird $\alpha_{1}=\alpha_{2}=\alpha_{6}=1$, $\alpha_{3}=10, \alpha_{4}=20$ und $\alpha_{5}=5$ gewählt. Beim optimierungsbasierten Entwurf nach (30) wird entsprechend Anmerkung 4 eine Mindestabklingrate von $\zeta=2$ festgelegt. Der optimierte Regler weist ein Gütemaß von 465,31 auf, während sich bei der händischer Auslegung ein Gütemaß von 836,11 ergibt. Während alle Regler dasselbe Führungsverhalten aufweisen, erkennt man in Bild 1 deutlich die geringeren Stellgrößenausschläge des optimierten Reglers. Bei $t=1 \mathrm{~s}$ tritt ein Führungsgrößensprung auf $w_{1}=10$ auf, nach $t=3 \mathrm{~s}$ ist zusätzlich $w_{2}=0,5$. Da die Eingangsgrößen im verwendeten Modell zu besseren Vergleichbarkeit skaliert sind, ist insbesondere die in Bild $1 \mathrm{zu}$ erkennende gleichmäßige Belastung der einzelnen Aktoren hervorzuheben.

Im Weiteren wird angenommen, dass die Matrizen $\boldsymbol{A}$ und $\boldsymbol{B}$ nicht mehr exakt bekannt sind. Sie lassen sich jedoch als (33) darstellen, wobei die Modellunsicherheiten dergestalt sind, dass eine starke Kopplung zwischen der Führungsgröße $w_{2}$ und dem Ausgang $y_{1}$ auftritt. In Bild 2 erkennt man, dass der zuvor entworfene Entkopplungsregler für das quadratische System zwar der Führungsgröße $w_{1}$ gut folgt, jedoch bei $t=3 \mathrm{~s}$ ein starker Einfluss aufgrund eines Führungsgrößensprunges auf $w_{2}=2$ herrscht. Für das quadratische System ergibt sich nach (37) die Schranke $\beta_{q}=3,47$. Der mittels des Verfahrens aus Abschnitt 4.2 optimierte Regler für das überaktuierte System weist demgegenüber mit $\beta_{\text {opt }}=1,42$ nach 20 Iterationen von Algorithmus 1 eine deutlich verringerte Kopplung auf. Beim Entwurf wurde dabei zusätzlich $\left\|\boldsymbol{G}_{\boldsymbol{u} \boldsymbol{w}}(s)\right\|_{\infty}$ begrenzt, um zu hohe Stellamplituden 
$\mathrm{zu}$ verhindern. Außerdem sind die Eigenwerte des geschlossenen Regelkreises entsprechend Anmerkung 6 auf einen Kreis um den Ursprung mit dem Radius $\varrho=9$ begrenzt. Die Optimierung erfolgte dabei wie im gesamten Beispiel mit Hilfe des LMI-Interfaces YALMIP [21] und des Solvers SeDuMi [31].

\section{Zusammenfassung}

In diesem Beitrag wurden zwei bekannte Entwurfsverfahren für lineare Entkopplungsregler auf den Fall überaktuierter linearer Systeme erweitert. Der Entwurf basierend auf der Vollständigen Modalen Synthese erlaubt eine anschauliche Interpretation der sich ergebenden zusätzlichen Freiheitsgrade. Die Erweiterung des Entwurfs nach Falb und Wolovich vereinfacht demgegenüber die gezielte Nutzung der Freiheitsgrade zur Minimierung der Stellenergie und zur Steigerung der Robustheit bezüglich unsicherer Modellparameter. Weitere Anwendungsgebiete von Entkopplungsregelungen für überaktuierte Systeme sind in der expliziten Berücksichtigung von Stellgrößenbeschränkungen sowie der Robustheit bezüglich exogener Störungen zu sehen.

\section{Danksagung}

Diese Arbeit wurde unterstützt von der Deutsche Telekom Stiftung (http://www.telekom-stiftung.de).

\section{Literatur}

[1] Arun Kishore, W. C.; Sen, S.; Ray, G.: Disturbance Rejection and Control Allocation of Over-Actuated systems. In: Proceedings of the IEEE International Conference on Industrial Technology. Mumbai, 2006, S. 1054-1059.

[2] Bernstein, D. S.: Matrix Mathematics. Princeton University Press, 2005.

[3] Boyd, S.; Ghaoui, L. E.; Feron, E.; Balakrishnan, V.: Linear Matrix Inequalities in System and Control Theory. SIAM, 1994.

[4] Chen, Y.; Wang, J.: A Global Optimization Algorithm for Energy-Efficient Control Allocation of Over-Actuated Systems. In: Proceedings of the American Control Conference. San Francisco, 2011, S. 5300-5305.

[5] Chilali, M.; Gahinet, P.; Apkarian, P.: Robust Pole Placement in LMI Regions. In: IEEE Transactions on Automatic Control 44 (1999), Nr. 12, S. 2257-2270.

[6] Cremer, M.: Festlegen der Pole und Nullstellen bei der Synthese linearer entkoppelter Mehrgrößenregelkreise, Teil 1. In: Regelungstechnik und Prozeß-Datenverarbeitung 21 (1973), Nr. 5, S. $144-150$.

[7] Cremer, M.: Festlegen der Pole und Nullstellen bei der Synthese linearer entkoppelter Mehrgrößenregelkreise, Teil 2. In: Regelungstechnik und Prozeß-Datenverarbeitung 21 (1973), Nr. 5, S. 195-199.

[8] Descusse, J.; La fay, J. F.; Malabre, M.: Solution to Morgan's Problem. In: IEEE Transactions on Automatic Control 33 (1988), Nr. 8, S. 732-739.

[9] Falb, P.; Wolovich, W.: Decoupling in the Design and Synthesis of Multivariable Control Systems. In: IEEE Transactions on Automatic Control 12 (1967), Nr. 6, S. 651-659.

[10] Galeani, S.; Serrani, A.; Varano, G.; Zaccarian, L.: On linear over-acutated regulation using input allocation. In: Procee- dings of the IEEE Conference on Decision and Control and European Control Conference. Orlando, 2011, S. 4771-4776.

[11] Herrera H., A. N.; Lafay, J. F.: New results about Morgan's problem. In: IEEE Transactions on Automatic Control 38 (1993), Nr. 12, S. 1834-1838.

[12] HärKEgARD, O.; GLAD, S. T.: Resolving actuator redundancy optimal control vs. control allocation. In: Automatica 41 (2005), Nr. 1, S. 137-144.

[13] Korn, U.; Zindler, K.: Vollständige modale Synthese robust stabiler Ausgangsvektorrückführungen unter Verwendung eines $\mathcal{H}_{\infty}$-Kostenfunktionals. In: at - Automatisierungstechnik 45 (1997), Nr. 6, S. 290-298.

[14] Koussiouris, T.: A frequency domain approach to the block decoupling problem. In: International Journal of Control 32 (1980), Nr. 3, S. 443-464.

[15] LiAo, F.; Lum, K.-Y.; WANG, J. L.: Constrained Control Allocation for Linear Systems with Internal Dynamics. In: Proceedings of the IFAC World Congress. Seoul, 2008, S. 3092-3097.

[16] Liv, G.P.; Ратton, R. J.: Parametric state feedback controller design of multivariable systems. In: International Journal of Control 61 (1995), Nr. 6, S. 1457-1464.

[17] Lohmann, B.: Vollständige Entkopplung durch dynamische Zustandsrückführung. In: at - Automatisierungstechnik 39 (1991), Nr. 9, S. 459-464.

[18] Lohmann, B.: Vollständige und teilweise Führungsentkopplung dynamischer Systeme durch konstante Zustandsrückführung, Teil 1. In: at - Automatisierungstechnik 39 (1991), Nr.9, S. 329-334.

[19] Lohmann, B.: Vollständige und teilweise Führungsentkopplung dynamischer Systeme durch konstante Zustandsrückführung, Teil 2. In: at - Automatisierungstechnik 39 (1991), Nr.9, S. 376-378.

[20] Lohmann, B.: Vollständige und teilweise Führungsentkopplung im Zustandsraum, VDI-Fortschrittberichte, Reihe 8, Nr. 244, Diss., 1991.

[21] LöFBERG, J.: YALMIP: a toolbox for modeling and optimization in MATLAB. In: Proceedings of the IEEE International Symposium on Computer Aided Control System Design. Taipei, 2004, S. 284-289.

[22] Meyer, C. D.: Matrix Analysis and Applied Linear Algebra. SIAM, 2001.

[23] Moness, M.; Amin, M.: Minimal-order precompensators for decoupling linear multivariable systems (A,B,C,E). In: International Journal of Control 47 (1988), Nr. 6, S. 1925-1936.

[24] Morgan, B.S.: The Synthesis of Linear Multivariable Systems by State-Variable Feedback. In: IEEE Transactions on Automatic Control 9 (1964), Nr. 10, S. 468-472.

[25] Oppenheimer, M. W.; Doman, D. B.; Bolender, M. A. Control Allocation for Over-Actuated Systems. In: Proceedings of the Mediterranean Conference on Control and Automation. Ancona, 2006, S. 1-6.

[26] Ostertag, E.: An Improved Path-Following Method for Mixed $\mathrm{H}_{2} / \mathrm{H}_{\infty}$ Controller Design. In: IEEE Transactions on Automatic Control 53 (2008), Nr. 8, S 1967-1971.

[27] Roppenecker, G.: On Parametric State Feedback Design. In: International Journal of Control 43 (1986), Nr. 3, S. 793-804.

[28] Roppenecker, G.; Lohmann, B.: Vollständige Modale Synthese von Entkopplungsregelungen. In: at - Automatisierungstechnik 36 (1988), Nr. 11, S. 434-441.

[29] Schrader, C. B.; Sain, M. K.: Research on system zeros: a survey. In: International Journal of Control 50 (1989), Nr. 4, S. $1407-1433$.

[30] Stefanovski, J.: Sufficient conditions for linear control system decoupling by static state feedback. In: IEEE Transactions on Automatic Control 46 (2001), Nr. 6, S 984-990.

[31] Sturm, J.F.: Using SeDuMi 1.02, a MATLAB toolbox for optimization over symmetric cones. In: Optimization Methods and Software 11 (1999), Nr. 1-4, S. 625-653. 
[32] Sva Ricek, F.: Zuverlässige numerische Analyse linearer Regelungssysteme. Vieweg \& Teubner, 1995.

[33] Voulgaris, P.; Valavani, L.: High performance linearquadratic and $\mathrm{H}$-infinity designs for a supermanuverable ${ }^{c}$ aircraft. In: Journal of Guidance, Control, and Dynamics 14 (1991), Nr. 1, S. $157-165$.

[34] Wei, M.; Wang, Q.; Cheng, X.: Some new results for system decoupling and pole assignment problems. In: Automatica 46 (2010), Nr. 5, S. 937-944

[35] ZACCARIAN, L.: Dynamic allocation for input redundant control systems. In: Automatica 45 (2009), Nr. 6, S. 1431-1438.

[36] Zhong, M.; Ding, S. X.; LAM, J.; WAng, H.: An LMI approach to design robust fault detection filter for uncertain LTI systems. In: Automatica 39 (2003), Nr. 3, S. 543-550.

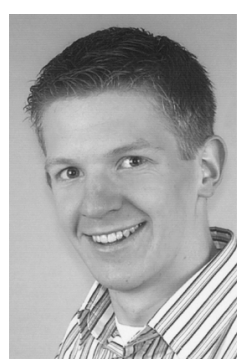

Dipl.-Ing. Arne Wahrburg ist wissenschaftlicher Mitarbeiter am Fachgebiet Regelungstheorie und Robotik im Institut für Automatisierungstechnik der Technischen Universität Darmstadt. Hauptarbeitsgebiete: Modellbasierte Fehlerdiagnose, Entkopplungsregelungen, Verteilte Systeme.

Adresse: Technische Universität Darmstadt, Institut für Automatisierungstechnik, Fachgebiet Regelungstheorie und Robotik, Landgraf-GeorgStr. 4, D-64283 Darmstadt, Tel.: +49-(0)6151-1670909, E-Mail: wahrburg@rtr.tu-darmstadt.de

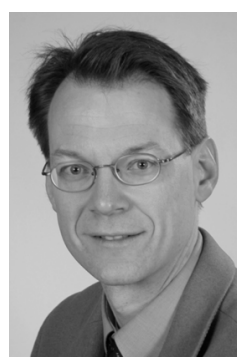

Prof. Dr.-Ing. Jürgen Adamy ist Leiter des Fachgebiets Regelungstheorie und Robotik im Institut für Automatisierungstechnik der Technischen Universität Darmstadt. Hauptarbeitsgebiete: Regelungsverfahren, Computational Intelligence, autonome mobile Roboter.

Adresse: Technische Universität Darmstadt, Institut für Automatisierungstechnik, Fachgebiet Regelungstheorie und Robotik, Landgraf-GeorgStr. 4, D-64283 Darmstadt, Tel.: +49-(0)6151-163442, E-Mail: jadamy@rtr.tu-darmstadt.de

Verfügbar unter

lediglich die vom Gesetz vorgesehenen Nutzungsrechte gemäß UrhG 